\title{
Major Players in Space, Quantum and Relativistic Theories
}

\author{
Jose Oreste Mazzini \\ oremazz@gmail.com
}

\begin{abstract}
Plank confine energy in quanta and later Heisenberg gave space-time limitations. Schrodinger wave function $\Psi$ express quantum oscillation and Born clarified this wave function $|\Psi|^{2}$ as the probability of presence. Hypothesis of space, interprets the wave function $\Psi$ as oscillatory space produced by the presence of quantized energy. Matter is confined in these quanta environment and its energetic space (quanta volume times its frequency) is related to surrounding space by Einstein equivalence principle. This theory develops a new way to interpret and integrate quantum mechanics with general relativity. Verifying the consistency of this quantumrelativistic interpretation, an explanation is given to the fundamental's ideas, observations and experiments of Modern Physics.
\end{abstract}

Keywords: theory of space, equivalent space flow, multidimensional space, quantum gravity, universe expansion.

\section{Introduction and First Thoughts}

Quantum Mechanics and Theory of Relativity are core developments in modern physics, but still there are many questions and joining points to be explained or solved. Physics still has this challenge, not only from scientific view, but also for the philosophical implications of nature quantum behavior.

From the quantum side, Max Born [1] great's interpretation gave the correct view that wave function $|\Psi| 2$ is related to physical probability of existence in a 1given space-time. Same as when nature is observed, aleatory behavior is found at quantum scale, but if more information is provided (as measurements), a reset of the cycle or a collapse of the system will happen. This measurement or additional information drastic change of quantum behavior, is the clue to consider imprecise values as basic existence on quantum systems. Probabilities of wave but observations of particles gave the well-known wave-particle duality; but wave can't explain why condense particle is always observed. Waveparticle duality is normally attached to only one entity with dual existence. Hypothesis approach this dilemma with two entities that coexists; space and energy (matter, electromagnetism, etc.). Energy will be present when space is present, even if some detailed physical value is limited; as expressed by the Uncertainty Principle. Having this concept, on each fluctuation, particle randomly acquires circumstantial valid-values that can be changed on each fluctuation. Unified values as total energy, total momentum, total charge, matter type, etc. are always preserved; physics core conservation principle. On Einstein's dices phrase, the answers will be in the details; dice with only one unique reality will be observed only one side at a time, constantly changing if no precise information is provided. This hypothesis ultra-fast aleatory behavior is the essence of quantum multiple solution; instantaneously superposition or coexistence is not considered. Philosophical implications as absolute deterministic nature or many world theory are sidelined.

Immediate question will be, how space has two concepts that oscillates? what happen when space-energy is not present? where does it goes? how conservation principle is preserved? Hypothesis considers that this space oscillation is between 3D space with no-space or nothing zone. Space wave function at nothing zone explains A) how conservation of total energy, total momentum, total charge, matter type, etc. is preserved at all time. B) How entangled particles can share information instantaneously; no-space provide no necessity to transport information neither overcome limitation of light speed. C) How particles change circumstantial values without a space transition. D) The zone where interaction between energy and other energies are shared. E) Big bang 3D space creation will be plausible explained as energy (always existing and conserved) coming from the nothing zone. By these assumptions, finally wave function $\Psi$ have a complete physical meaning and many quantum rareness overcome; quantum observations of random observations, tunneling, entanglement and double slit experiment are understandable nature behavior under this Theory of Space.

From Relativistic side, Einstein [2] gave the wonderful relationship that mass is a manifestation of energy and change completely the view of a rigid space. Energy carrying a length contraction and time dilation of spacetime. Later on his glorious General Relativity [3], he showed that one energetic source was curving physical space-C*time and generating the gravitation effect over other energetic sources. Space-C*time completely variable over 3D space having implicit local length and time own scale all over the observable space. Careful is recommended when space is joined with time dimension, the connection with 3D space is with length $C^{*}$ time and not only time. Difficult to imagine the joint of space and time, two different dimensions; meanwhile is understandable joining coordinates between $3 \mathrm{D}$ length with another length, the energy wave length $\lambda$ Energy $=\mathrm{C} *$ time. Here the length and time used is the existence length-time of the quantum package cycle $(\lambda \&$ $1 / v)$, farther distances are ruled by the diffuse influence of energy over its surroundings.

Special Relativity interrelates energy given by speed with space length, that is, an energy increment with time scaledilation and length scale-contraction; Space and time Relative-Relativity to Energy. Note that this implies that kinetic energy is an absolute value and not relative to the observer; conservation of energy is a must, energy is not 
created by playing with imaginary moving frames. Twins' paradox solution implies that only one twin is younger, the one that really gets kinetic energy. Lorentz frame relation is only applicable on real physical frames, imaginary moving frames can mislead to speed greater than C. Speed is incremented by absorbing energy or lowering speed by giving energy; in quantum dimensions, this will imply a photon absorption (increment-change of momentum) or an emission (lowering its energy). Below on this paper, for just imaginary moving frames, a relation between mass and kinetic energy is presented in such way that an apparent kinetic energy increment implies a mass energy decrements and vise verse, total energy is preserved and not depends on moving frame.

It is difficult to visualize 4D space as a curved coordinate of space-time, a simpler way is to change local scale. The result is unbent coordinate axis with variable scale depending on the presence of energy; a dependence between dimensional 3D length with energy length is contained. Our mind will be imagining in the usual cubic 3D space and the energy will be indirectly present by means of variable local scale on each coordinate.

A common way to visualized General Relativity attraction is using a elastic sheet with different heavy spheres; this mislead curved space-time because gravity is used to explain gravity. This example won't work at International Space Station on space, even if elastic sheet is conveniently deformed.

An equivalence dynamic space is more proper to think of General Relativity, Einstein imaginative equivalence principle [4] began with a man having an equivalent space flow in acceleration that continuously pull him down; not only an equivalent force is deduced but also and equivalent time dilation (as applied for GPS corrections). By this equivalence, hypothesis relates the kinetic energy of only one axis to the whole package internal space; as a consequence of this, energetic volume of the package affects its surrounding $3 \mathrm{D}$ space in a diffusion way $\left(1 / \mathrm{R}^{2}\right)$. Moving equivalent space flow that ends joining one energy source with others; like quantum gray holes sucking space and attracting surrounding energy. Space flow without energy consumption or friction-less equivalent flow as seen on the extensive propagation of gravitational wave. Compressible equivalent flow or dimensional contraction by energy (mass and momentum) as seen on Special Theory of Relativity. Formulation is the same, but the concept of vacuum-space as a friction-less fluid flow will have not only a macroscopic explanation, but also implies a limit over the quantum package dimension; Liberati and Maccione [5] gave that approach.

The presentation of this paper is divided first to the oscillation of 3D-space with Nothing-space and its similarity to the wave function $\Psi$ with complex number managing space fluctuation. Space presence getting a probabilistic space distribution of existence; since this space coexist with matter, i.e., particles will also have that same probabilistic distribution. Then, this hypothesis is submitted to explain fundamentals observations and deductions of Modern Physics. Using the equivalent space flow calculations, the
Newtonian law of attraction is obtained and QPA is deduced (Quantum Package Average Area QPA transverse to the speed direction). With this, Newton attraction law is expanded with relativistic considerations when energy is very near to other ones. From special relativity and Minkowsky invariance the 4th dimension is given as an energy length dimension with many subdivided dimensions. Finally, gravitation attraction-repulsion is deduced and taken into consideration for the expansion of the universe. All the effort in validating this Theory of Space is completely worthwhile.

\section{Hypothesis Part 1}

\section{D-vacuum space and No-space}

Theory of Space considers that energy is confined on 3D space inside the quantum package; space oscillation that on each cycle, space is present and absent $\mathrm{n}$ times in accordance to its energy level. This internal energy-space is related to the $3 \mathrm{D}$ surrounding space outside the quantum package by the equivalence volume flow. Even more, quantum energy is related to other dimensional space as shown on the second part of this hypothesis. Considering 3D quantum space flow plus multidimensional space energy gives additional sustain to this Theory of Space.

Inside the quantum package, its space oscillates by sectors, in a proportion given by existence plane wave, obtaining an expectancy of presence same as the probability density of the well-known wave function $\Psi$. Quantum space changes from Full presence $(100 \%)$ at the beginning to $100 \%$ missing space or no-space or nothing zone (same as observed on vacuum space phenomena as virtual particles) and with same inertial gradualism returns to a new full space. Quantum space involved is related to outside surrounding space in an equivalent $3 \mathrm{D}$ space flow like a vibrating gray hole at quantum dimension. Inclusive, black holes can be considered gigantic quantum package sucking equivalent $3 \mathrm{D}$ space at $\mathrm{C}$ speed in its frontier.

This assumption maintains existence and energy over time, even at nothing zone. Another implication is that time is common and continuous in both scenarios (space zone and nothing zone) so wave is applicable. A third implication is that no diffuse or partial presence of particles, they are at space presence zone or at nothing zone; progressively alternation is only between $3 \mathrm{D}$ space vs nothing zone, duality is understood as compact particle coexisting within its wavy package presence. A fourth implication is that losing its 3D quantum space, some physical variables (without measurement nor nature definition) are undefined, giving explanation to nature randomness behavior or multiple coexistence solutions; one solution after each passage over nothing zone, multiple cycles imply existence of multiple solutions. A fifth implication is that moving package must have a volume share between its position of one cycle vs the following one, particles can't return from nothing into a zone that no longer correspond to package new 3D position; because of this, package speed will have the speed limit of $\mathrm{C}$.

Assumption that can be imagine with a quantum package volume describe by the energetic wave length multiplied by 
its transverse area QPA (Quantum Package Area). The volume per unit time involved in a cycle will be given by $n$ $* \lambda$ Energy $* \mathrm{QP} \mathrm{A} * v$. For $\mathrm{n}$ greater than 1 , cycle package will be $n$ times smaller packages vibrating at $n * v$; that is, greater energy level will involve greater quantum volume per unit time.

Space presence will be expressed in a similar way of wave function $|\Psi| *|\Psi|$ with a rate given by its energetic frequency $\mathrm{n} * v$. This space oscillation implies no diffuse particle, nor superposition-coexistence of multiples valid values. Just a particle with physical values in correspondence to its 3D space existence; it is or it isn't situation. All calculations of particle behavior and interactions are statistical and noncontinuous but always involving system valid-values with the absolute prevalence of total energy, total momentum, total charge and matter type at nothing passage.

\section{Interpretations}

After checking the consistency of this space interpretation, an explanation is made to some of the fundamental observations of modern physics.

\subsection{Interference}

\section{Double slit experiment}

The famous double slit experiment done by Claus Jonsson [6] on electrons, reveals the behavior of mass over its space; normally interpreted as interference of waveparticle duality. From light interference, the correct slit width and separation is crucial for having interference. In the electron case, the wave length corresponding to its mass energy is way off the slit width of the experiment. Something is producing the interference with wave length much greater than compact particle mass wave length.

Hypothesis considers that the fluctuating space wave is the one compromise at the double slits, the $3 \mathrm{D}$ space zone of one slit propagation, does the destructive interference with nothing zone, of the other slit propagation. Space is divided in two solutions that continues its propagation obtaining a constructive-destructive interference. Individual electrons on this experiment shows no diffuse electron at the end, just one compact electron each time following the interference pattern of its combine space propagation. No mixture between diffuse electron nor superposition of multiple solution at a given time. Wave function of the space going through double slit creates an interference of quantum space possible trajectory and not the particle; particles existence is possible only where its coexisting space is possible. Particles with their potential energy, kinetic energy, spin, charge, mass, will follow the system valid values on new quantum space on every fluctuation of the existence cycle. When a detection of passage is done over one slit, then a reset of its cycle is assumed and propagation continues only from this specific slit; no longer existence of two slit solutions, so no interference is expected neither observed.

\subsection{Cycles reset}

\section{Polarized photons and Bell's inequality}

On polarized photons, if a filter is applied with phase angle $\beta$ between them, the probability of photon existing after this filter is $\cos ^{2}(\beta)$ and for not being is $\sin ^{2}(\beta)$. If a second intermediate polarized filter is included at $\alpha$ angle from incoming polarization, a less destructive interference or higher probability of existence is obtained at the end. Inertial gradualness of space wave will change less by steps resets than if nature is left alone all the way through up to the final event. With $0<\alpha \leq \beta \leq \pi / 2$, the probability of not being at the end, will be:

$\sin ^{2}(\alpha)+\cos ^{2}(\alpha) * \sin ^{2}(\beta) \geq \sin ^{2}(\beta)$ withoutreset

$\sin ^{2}(\alpha)+\cos ^{2}(\alpha) * \sin ^{2}(\beta-\alpha) \leq \sin ^{2}(\beta)$ withreset

when $\beta=2 * \alpha$ the probability with reset will be:

$\sin 2(\alpha)+\cos ^{2}(\alpha) * \sin ^{2}(\alpha) \leq \sin ^{2}(2 \alpha)$

Equation (1) shows inequality where second filter non-pass probability is maintained; same case is seen on logic of Venn diagram, but this inequality isn't observed on this case. Photons once passes a filter its polarized information is given by the last passage and not the initial condition; this confirms that the cycle have been reset or system conditions have change after a passage. Same case of double slit experiment, when electron passage over a slit is observed, immediately the system will be reset.

Additionally, if more than one particle follows a synchronize fluctuation of cycles, perfect correlation will happen and this will be the interpretation of entangled particles, even more, when on one of them some value resets or collapse, the other particles will immediately change to proper valid-value. A common meeting at nothing zone will give harmonic value in correspondence to core conservation laws. Since nothing zone doesn't contains space, there is no speed limitation $C$ proper of having space traveling and this reconfirms the non-locality of the system. Spooky action that Bell's inequality [7] cleverly determine the reality of nature; no locality requirement nor hidden value. Inequality being challenge is more understandable with this example of Venn diagram having logic sets arranged in $\mathrm{A}, \mathrm{B} \& \mathrm{C}$ measurement sets in such a way that $\mathrm{P}\left(\mathrm{A} \cap \mathrm{B}^{\prime}\right)+\mathrm{P}\left(\mathrm{B} \cap \mathrm{C}^{\prime}\right) \geq$ $\mathrm{P}\left(\mathrm{A} \cap \mathrm{C}^{\prime}\right)$ where $\cap$ is the information simultaneously obtained from the experiment on the other entangled particle (sets A' , B'\& C' ). Note that this logical inequality doesn't apply when entangled particle involved have partial reset of their cycle.

Any measurement over one entangled particle will also reset the other entangled particle, even with no measurement nor any other nature conditions is applied. This is the key Bell used to distinguish hidden value from quantum entanglement; connected existences assuming its value at a distance. Using phase of 90 degree and in between 45 degree gave an excellent experimental condition.

\subsection{Tunneling}

Quantum tunneling brightly noticed by Friedrich Hund [8] in 1927 and explained mathematically by George Gamow and independently by Ronald Gurney - Edward Condon, both explaining tunneling by the probability density of existence even outside a potential wall. How can a particle pass through a potential wall, even if there is some probability that sustains it? Hypothesis considers that particle at the nothing zone has no 3D space nor spatial limitation, probabilistic appearance on any position on replacing space is a compressible and obligatory deduction. No conflict with 
3D space walls; even more, atomic orbital nodes have solutions that separates orbital in regions, this jumps between them will also be understood with the same reasoning. Particle's presence-cycle changing $\mathrm{n}$ times, will pass over nothing zone $\mathrm{n}$ times, changing position, direction and spin $\mathrm{n}$ times; higher energy has more probabilities of adopting an unexpected position than lower energy particles. Once the particle overcomes the wall a reset of its cycle is assumed as happens when photons pass a polarized filter.

\subsection{Cycle existence}

\section{Heisenberg uncertainty principle}

Total energy and total momentum are completely assumed at $100 \%$ nothing passage when position and movement are already missing ( $0 \%$ presence in manifest $3 \mathrm{D}$ space); this interprets the powerful Heisenberg [9] on the uncertainty principle $\Delta \mathrm{p} * \Delta \mathrm{r}=\mathrm{h}=\Delta \mathrm{E} * \Delta \mathrm{t}$; packaged existence by limiting simultaneous definition of momentum and position / energy and time. Energy in quanta of action h conditioned to a given time $1 / v E ; E=h v E$. Another way to express is to multiply both sides of Heisenberg equation by light speed, $E$ $* \lambda \mathrm{E}=\mathrm{hC}=$ constant; bigger energy implies less wave length for the package action $\mathrm{h}$. Momentum, in same quanta, with the given space $\lambda \mathrm{K}, \mathrm{pC} * \lambda \mathrm{K}=\mathrm{hC}=\mathrm{constant}$; more dynamic energy implies less dynamic wave length, or $p=h / \lambda K$ (see De Broglie on next section).

Quanta containing total energy and total momentum in an oscillating space at a given frequency, this is the essence of quantum package and existence cycle; existence must be packet. Other circumstantial values assume a valid-value of the system with no previous conditioning. Circumstantial values that are constantly changing depending on position, kinetic-momentum, spin-momentum, potential energy, etc. That is assuming on every fluctuation; a circumstantial value because its undefinable detailed existence. Defined or collapsed events will reset the cycle and condition the particles on next 3D space.

\subsection{Special Relativity and Minkowski space}

From De Broglie [10] impressive relation of $p=h / \lambda$ and Einstein [2] wonderful Special Theory of Relativity where mass is an energy manifestation $\mathrm{M}^{\prime}=\mathrm{E} / \mathrm{C}^{2}$; light speed $\mathrm{C}$ as universal speed limit, inertial mass increment due to inertial energy increment $M^{\prime}=\gamma$ Mo and 4th dimension as a spacetime concept. Both contributions to modern physics are interrelated by their energy nhv $=\gamma \mathrm{MoC}^{2}$. Minkowski [11] important invariance relation $\left(\mathrm{x}^{2}+\mathrm{y}^{2}+\mathrm{z}^{2}\right)-\mathrm{C}^{2} \mathrm{t}^{2}$ consolidates the time relation to 3 -dimensional space. Hypothesis of energy space dimension considers the same, but not any time nor any length, the quantum time $\left(v^{-1}\right)$ involved in one existence cycle with speed $\mathrm{C}$ and the length of the quantum package mayor axis. That is the length of energetic particlephoton, so i $\mathrm{Ct}=\mathrm{i} \lambda \mathrm{E}(\lambda \mathrm{EvE}=\mathrm{C})$ and invariance in the package is $\Delta \mathrm{r}^{2}-\lambda \mathrm{E}^{2}$. More understandable because the frame of reference is where the energy is; diffused importance has any other time and any dimension all over the moving frame; energy locality interrelated with its local 3D space and gradually decrease over its surrounding 3D space. This implies that surrounding space will also suffer a length contraction and time dilation. So, 4th dimension, energylength, related to observable 3-dimensional length; this joins energy with space contraction $r \gamma^{-1}$, time dilation $t \gamma^{-1}$ and mass increment $\gamma \mathrm{Mo}$ (see Figure 1).

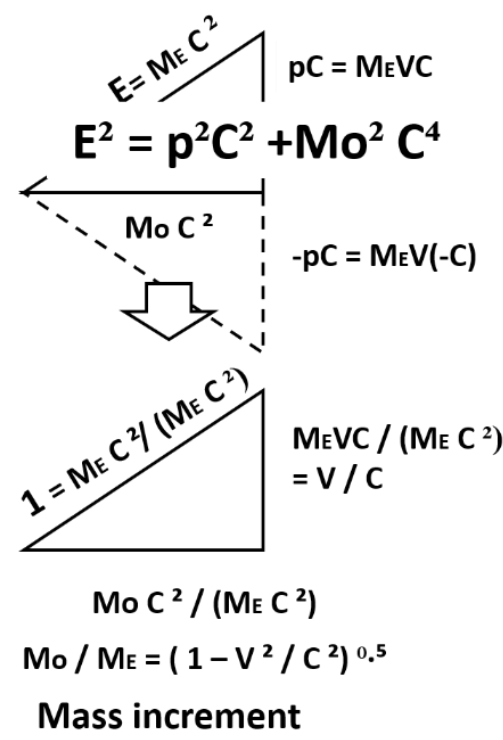

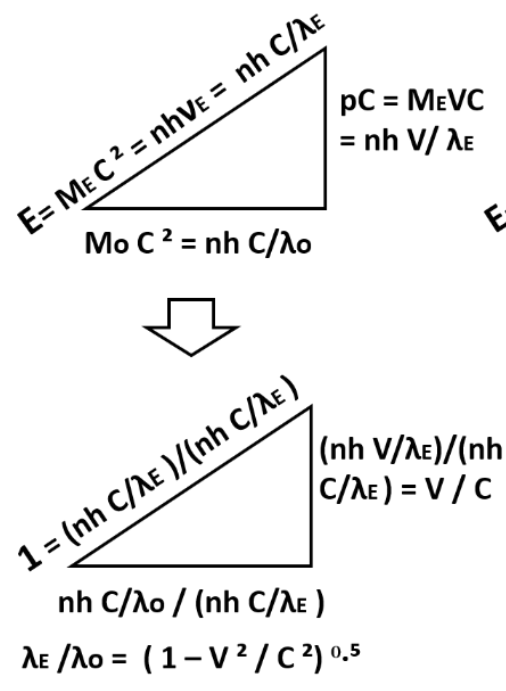

Length contraction

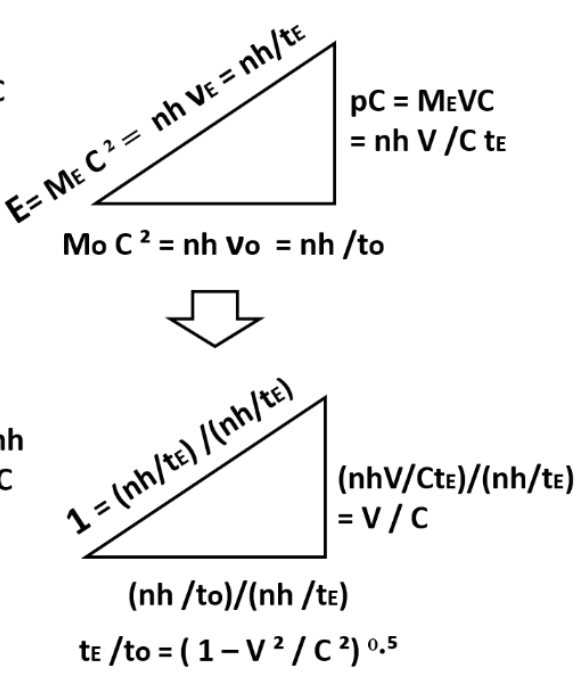

Time dilation

Figure 1: Energy space, four dimensions invariance; inertial mass increment, length contraction, time dilation. Lower triangles have hypotenuse $=1$, at scale of top triangles; vertical side is V/C and using Pythagoras the other side is Lorentz expression.

The Special Theory of Relativity reinforces the core principle of conservation of energy. In the case of a particle going at a speed $\mathrm{V}$, it contains a kinetic energy plus a mass energy, but from the view of the particle itself, there will be no kinetic energy, just a mass energy perfectly incremented and balanced by Lorentz $\gamma$ factor [12]. Even if the speed $\mathrm{V}$ is a relative observation, it will be assuming an apparent kinetic energy increment with its respective mass wavelength increment, that is, diminishing its relativistic mass by $\mathbf{M}^{\prime} \gamma^{-\mathbf{1}}$; total energy is conserved. Meanwhile, the 
inertial frame at the particle will consider no kinetic energy, its energetic mass 9 compensates by increments on Mo $\gamma$. No real energy change in this relativistic view of speed $\mathrm{V}$, just an invariant view between kinetic plus mass energy achieving a full conservation of energy. By this, energy interrelates with space and hypothesis relates oscillatory space within its package volume; note that oscillation is energy as M. Planck [13] remarkable founder equation of Quantum Theory expressed as $\mathrm{E}=\mathrm{nh} v \mathrm{E}$.

\subsection{Schrodinger and wave function}

Relativistic invariance involves the negative relation between the wavelength of all types of energy versus 3D space, this is because when energy increments, its wavelength contracts in the same way as 3D space does; contrary to other invariances that one diminishes meanwhile the other increments. Again, useful irrational number $i=\sqrt{ }$ -1 is applied by E. Schrodinger [14] when he related the amazing wave function $\Psi$ on space real number axis with energy and momentum at irrational number axis; math of complex number properly applied. Reasoning that mass is in the same moment and place where the particle is $(\underline{M}=M o \Psi$, $\underline{\mathrm{x}}=\mathrm{x} \Psi)$. Again, reasoning that unified energy and unified momentum are in the place where the particle is, only 900 out of phase; different types of energy and momentum are on phase, but total energy and total momentum are wave function at the irrational axis $(\underline{E}=\mathrm{i} \sim \partial \Psi / \partial \mathrm{t}, \mathrm{p}=-\mathrm{i} \sim \partial \Psi / \partial \mathrm{r})$. Therefore, unexplained $\Psi$ express nature oscillating numbers phenomena, when the real number diminishes, irrational number increments and vice versa; with inertial behavior proper of plane waves and indirectly accordance with conservation principle. Normally, oscillation implies interchange, one part loosing and the other acquiring and vice versa. SchrodingerBorn wave oscillation are probabilistic existence of particle-values, but questions where unanswered; what does the irrational energy, momentum, charge, mass, mean? Why space zones are with minimum or no probability? How an electron can jump between orbits that are separated? Why nature behaves in aleatory way? How does multiple solutions can coexist? Hypothesis gives this irrational numbers a proper zone of nature, the nothing zone shared with manifest 3D space existence; math of complex numbers and Hilbert space is perfectly applicable. Core physical values are preserved as conservation laws and symmetry express, so well demonstrated by Noether theorem [15].

$$
\begin{gathered}
|\Psi|^{2}=|\Psi| *|\Psi|=[\cos (\omega)-\mathrm{i} \sin (\omega)] *[\cos (\omega)+\mathrm{i} \sin (\omega)]= \\
\cos ^{2}(\omega)+\sin ^{2}(\omega)=1
\end{gathered}
$$

Note that plane waves beside having inertial change observed on nature, also have a mathematical goodness; when derivative is applied, wave is maintained just changing its phase position by 90 degrees; now quantum energy operator is understandable containing $\mathrm{i}$ (rotating from irrational axis to real axis), the $-\partial \Psi / \partial \mathrm{t}$ (imaginary energy increments when particle presence decrements; $\sin (\omega)=-\partial$ $\cos (\omega) / \partial \mathrm{t})$ and $\sim$ amount time-energy per fluctuation. Hypothesis considers wave function $\Psi$ as the proper math for handling space fluctuation.

\section{Hypothesis Part 2}

\section{Space - Energy}

Same as the equivalence principle of Einstein [4], a particle on equilibrium (no acting forces) suffers an equivalent space movement-flow attraction in correspondence to the presence of energetic volume flow of other particles, its equivalent space acceleration breaks equilibrium and generates a movement unless a force is applied to maintain its position. In this way, internal quantum space is related to outside surrounding space in diffuse way; diffusion that implies not only an equivalent accelerated space (quantum gravity) but also an equivalent speed that provides length contraction and time dilation. Many types of energy, kinetic, mass, potential, electromagnetic field, etc.; all of these, beautiful interrelated energies, act as one over 3D space (4th total energy dimension).

\subsection{Equivalent space flow or Quantum Gravity}

From the classical gravitational laws expressed by Isaac Newton laws [16] in 1687, the attraction force of mass 1 and mass 2 separated by the distance $R$ is given by $G * M 1$ $* \mathrm{M} 2 / \mathrm{R}^{2}$ where $\mathrm{G}=6.67430(15) \times 10-11 \mathrm{~m} 3 \mathrm{~kg}^{-1} \mathrm{~s}-2$. On the hypothesis of equivalence space flow, the energy of one mass generates an apparent suction of space towards it, the other nearby mass moves because of this equivalence space acceleration and vice versa, second mass generates the same effect to the first mass. The amount of equivalent energetic space is proportional to the wave length $\lambda$ Energy $* \mathrm{QP}$ A of the quantum package, to the quantum number $\mathrm{n}$ and to the number of events given by its vibrating frequency; where QPA is the transverse Quantum Package Average Area. This energetic space affects the surrounding in the diffuse way $1 / R^{2}$. Having this, the following formulation is developed for obtaining the equivalent speed $\mathbf{S}$ and equivalent acceleration A perceive by other energetic points at a distance Rfrom the first one.

$$
\begin{aligned}
& \mathbf{S}_{1}=\mathrm{n}_{2} * \lambda \mathrm{E}_{2} * \mathrm{QPA} * v_{2} /\left(4 \pi / \mathbf{R}^{2}\right) \\
& \mathbf{A}_{1}=\mathrm{n}_{2} * \lambda \mathrm{E}_{2} * \mathrm{QPA} * \mathrm{v}_{2}{ }^{2} /\left(4 \pi \mathbf{R}^{2}\right)
\end{aligned}
$$

Considering $4 \pi R^{2}$ as the effect reduction of the equivalent space flow in all directions due to quantum space over the distance $R$ between packages 1 and 2 .

QPA is deduce from Newtons brilliant equation $\mathbf{F}_{1}=\mathbf{M}_{1} * \mathbf{A}_{1}$ where $\mathbf{A}_{1}$ is taken from equations (6) identical reasoning for $\mathbf{A}_{2}$ by attraction of $\mathrm{M}_{1}$. Also, $\mathrm{M}_{2} \mathrm{C}^{2} \approx \mathrm{n}_{2} \mathrm{~h} * v_{2}$ and $\mathrm{M}_{1} \mathrm{C}^{2} \approx \mathrm{n}_{1} \mathrm{~h}$ $v_{1}$ for particle small speeds and for equivalent space flow small speed; Mass at rest energy > Kinetic energy:

$$
\begin{gathered}
\mathrm{G} * \mathrm{M}_{2} / \mathbf{R}^{2} \approx \mathrm{n} 2 * \lambda \mathrm{E}_{2} * \mathrm{QPA} * v_{2}^{2} /\left(4 \pi \mathbf{R}^{2}\right) \\
\mathrm{QPA} \approx 4 \pi \mathrm{G} \mathrm{M} /\left(\mathrm{n}_{2} \mathrm{C} v_{2}\right) \\
\mathrm{QPA} \approx 4 \pi \mathrm{n}_{2} \mathrm{G}\left(\mathrm{M}_{1} * \mathrm{C}^{2}\right) /\left(\mathrm{n}_{2} * \mathrm{C}^{3} * v_{2}\right) \\
\mathrm{QPA} \approx 4 \pi \mathrm{G}\left(\mathrm{n}_{2} \mathrm{~h} * v_{2}\right) /\left(\mathrm{n}_{2} * \mathrm{C}^{3} * v_{2}\right) \\
\mathrm{QPA} \approx 4 \pi \mathrm{Gh} / \mathrm{C}^{3}
\end{gathered}
$$

$\mathrm{QPA} \approx 4 \pi \mathrm{hG} / \mathrm{C}^{3}=4 * 3.141592 * 6.62607 \times 10-34 *$ $6.67430 \times 10-11(2.99793 \times 10+8) 3 \approx 2.06256 \times 10-68[\mathrm{~m} 2]$ (12) 
QPA can also be expressed as $\pi \mathrm{r}^{2}$ QPA where rQPA $=\sqrt{ }$ $4 \mathrm{hG} / \mathrm{C}^{3}=8.1027 \times 10-35[\mathrm{~m}]$ near Planck unit length. Having QPA and equation (6), attraction Force of mass 1 will be the equivalent space flow from the oscillatory energy of mass 2:

$$
\begin{gathered}
\mathbf{F}_{1}=\mathrm{M}_{1} \mathrm{n}_{2} \lambda \mathrm{E}_{2} 4 \pi \mathrm{Gh} * \mathrm{v}_{2}^{2} /\left(\mathrm{C}^{3} 4 \pi \mathbf{R}^{2}\right) \\
\mathbf{F}_{1} \approx \mathrm{M}_{1} \mathrm{G}\left(\mathrm{n}_{2} \mathrm{~h} \lambda \mathrm{E}_{2} \mathrm{v}_{2}^{2}\right) /\left(\mathrm{C}^{3} \mathbf{R}^{2}\right)=\mathrm{M}_{1} \mathrm{G}\left(\mathrm{n}_{2} \mathrm{~h} v_{2} \mathrm{C}\right) /\left(\mathrm{C}^{3} \mathbf{R}^{2}\right) \\
\mathbf{F}_{1} \approx \mathrm{M}_{1} \mathrm{G}\left(\mathrm{M}_{2} \mathrm{C}^{2} \mathrm{C}\right) /\left(\mathrm{C}^{3} \mathbf{R}^{2}\right)=\mathrm{G} * \mathrm{M}_{1} \mathrm{M}_{2} / \mathbf{R}^{2}
\end{gathered}
$$

Deducing Newton mass attraction law by the appropriate QPA factor; a very intuitive way that associates energy with proper quantum volume and its surrounding diffuse influence. On very near masses the space flow will be at very high-speed giving a Lorentz effect over its mass by factor of $\gamma_{1}=1 / \sqrt{ }\left(1-V_{1}{ }^{2} / C^{2}\right)$; the extension of equation(14) will be:

$\mathbf{F}=\mathrm{G} \mathrm{n} \mathrm{n}_{1} \mathrm{~h} v_{1} \mathrm{n}_{2} \mathrm{~h} v_{2} /\left(\mathrm{C}^{4} \mathbf{R}^{2}\right)=\mathrm{G} \sqrt{ }\left[\left(\mathrm{Mo}_{1}{ }^{2} \mathrm{C}^{2}+\mathrm{p}_{1}^{2}\right)\left(\mathrm{Mo}_{2}{ }^{2} \mathrm{C}^{2}+\right.\right.$ $\left.\left.\mathrm{p}_{2}^{2}\right)\right] /\left(\mathrm{C}^{2} \mathbf{R}^{2}\right)(16)$

where $\mathrm{p}_{1}=\mathrm{Mo}_{1} \gamma_{1} \mathrm{~V}_{1}$ and $\mathrm{p}_{2}=\mathrm{Mo}_{2} \gamma_{2} \mathrm{~V}_{2}$.

$\left.\mathbf{F}=\mathrm{G} \sqrt{ }\left[\mathrm{Mo}_{1}{ }^{2} \mathrm{C}^{2}-\mathrm{Mo}_{1}{ }^{2} \mathrm{C}^{2} \mathrm{~V}^{2} / \mathrm{C}^{2}+\mathrm{Mo}_{1}{ }^{2} \mathrm{~V}_{1}{ }^{2}\right) \gamma_{1}{ }^{2}\right] * \sqrt{ }\left[\left(\mathrm{Mo}_{2}{ }^{2} \mathrm{C}^{2}\right.\right.$

$\left.\left.-\mathrm{Mo}_{2} \mathrm{C}^{2} \mathrm{~V}_{2}^{2} / \mathrm{C}^{2}+\mathrm{Mo}^{2} \mathrm{~V}_{2}^{2}\right) \gamma_{2}^{2}\right] /\left(\mathrm{C}^{2} \mathbf{R}^{2}\right)$

$\mathbf{F}=\left(\mathrm{G} \gamma_{1} \gamma_{2}\right) \mathrm{Mo}_{1} \mathrm{Mo}_{2} / \mathbf{R}^{2}$

(18)

where Mo1, Mo2 are mass value at rest and $\gamma 1, \gamma_{2}$ are their equivalent relativistic factor. Newton's attraction constant $G$ is incremented on very near masses.

Now the concept of space-time is a little more understandable; Energy presence (kinetic, mass, electric, etc.) is confined on quantum space, and this space creates a equivalent surrounding flow of space, so space flow acceleration is gravitation effect and space flow speed gives a correction factor to constant $G$.

\subsection{Multidimensional space}

Hypothesis energy space relation $\left(\lambda_{\mathrm{e}}\right)$ involves that kineticmomentum energy can have two possible arrays $\pm \lambda_{\mathrm{k}}$, besides the other two arrays of mass energy $\pm \lambda \mathrm{M}$; solution $1: 1 / \lambda_{\mathrm{e}}=$ $\mid \rightarrow 1 / \lambda_{\mathrm{k}}+\rightarrow 1 / \lambda$ Mo $\mid$, solution $2: 1 / \lambda_{\mathrm{e}}=\mid \leftarrow 1 / \lambda_{\mathrm{k}}+\rightarrow 1 / \lambda$ Mo |, solution 3: $1 / \lambda_{\mathrm{e}}=\mid \rightarrow 1 / \lambda_{\mathrm{k}}+\leftarrow 1 / \lambda$ Mo $\mid$, solution $4: 1 / \lambda_{\mathrm{e}}=\mid$ $\leftarrow 1 / \lambda_{\mathrm{k}}+\leftarrow 1 / \lambda$ Mo $\mid$. Energy dimension orientation $\left(\lambda_{\mathrm{e}}\right)$ with respect to $3 \mathrm{D}$ space giving 4 arrays or solutions as Dirac [17] showed on the creative relativistic equation. From $\mathrm{E}^{2}=$ $\mathrm{p}^{2} \mathrm{C}^{2}+\mathrm{Mo}^{2} \mathrm{C}^{4}$ that is $\pm \mathrm{pC}, \pm \mathrm{MoC}^{2}$; four arrays as seen on Dirac relativistic equation

$$
\mathrm{E}^{2} \mathrm{C}^{2}-\mathrm{p}^{2}-\mathrm{Mo}^{2} \mathrm{C}^{2}=0
$$

$$
\left(\mathrm{i} \hbar \gamma^{0} \partial \lambda+\mathrm{i} \hbar \gamma^{1} \partial \mathrm{x}+\mathrm{i} \hbar \gamma^{2} \partial \mathrm{y}+\mathrm{i} \hbar \gamma^{3} \partial \mathrm{z}\right) \boldsymbol{\Psi}-\mathrm{MoC} \boldsymbol{\Psi}=0
$$

Energy orientation is determined by mass orientation $\rightarrow \lambda \mathrm{E}=$ $\gamma^{-1}(\rightarrow \lambda \mathrm{Mo})$, matter and antimatter. The kinetic-momentum energy oriented interpreted as spin oriented; having both alternatives $\rightarrow \lambda_{\mathrm{k}}= \pm(\rightarrow \lambda \mathrm{Mo}) \beta^{-1} \gamma^{-1}$ by De Broglie equation $\mathrm{p}=\mathrm{h} / \lambda_{\mathrm{k}}$. Where Total energy contains Kinetic energy orthogonal to Mass energy.

$$
1 / \lambda_{\mathrm{e}}^{2}=1 / \lambda_{\mathrm{k}}{ }^{2}+1 / \lambda \mathrm{Mo}^{2}=1 / \lambda \mathrm{Kx}^{2}+1 / \lambda \mathrm{Ky}^{2}+1 / \lambda \mathrm{Kz}^{2}+1 / \lambda \mathrm{Mo}^{2}
$$

$\lambda \mathrm{Kx}=\lambda_{\mathrm{e}} \beta \mathrm{x}^{-1}=\lambda$ Mo $\gamma^{-1} \beta \mathrm{x}^{-1}$ where $\gamma$ is Lorentz contraction, $\beta \mathrm{x}=\mathrm{Vx} / \mathrm{C}$; same for $\mathrm{y}$ and $\mathrm{z}$ axis.

$\lambda_{\mathrm{e}}=\left[1 / \lambda \mathrm{Kx}^{2}+1 / \lambda \mathrm{Ky}^{2}+1 / \lambda \mathrm{Kz}^{2}+1 / \lambda \mathrm{Mo}^{2}+\ldots\right]-1 / 2$

$=\left[\gamma^{2} \beta \mathrm{x}^{2} / \lambda \mathrm{Mo}^{2}+\gamma^{2} \beta \mathrm{y}^{2} / \lambda \mathrm{Mo}^{2}+\gamma^{2} \beta \mathrm{z}^{2} / \lambda \mathrm{Mo}^{2}+1 / \lambda \mathrm{Mo}^{2}+\ldots\right]-$ $1 / 2$

Note that i $\mathrm{Ct}=\mathrm{i} \lambda \mathrm{E}$, 4th dimension can be expressed as $4 \mathrm{th}$, 5 th, and more dimensions depending on expressing total energy or the individual types of manifest energy involved (Kinetic-momentum, spin-momentum, mass energy, potential energy, weak force energy, strong force energy, etc.) that is energy multidimensional space. As seen on the intuitive 5D of Theodor Kaluza [18] towards a unified theory.

\subsection{Negative solutions: Spin and antimatter}

Kinetic-momentum energy expresses absorption or emission of electromagnetic photon on changing speed and/or direction as part of momentum interaction. The hypothesis of Spin as a particular type of momentum in quanta package that interacts over surrounding magnetic fields. Note that orientation follows the circumstantial position behavior, that is, spin adopting an orientation on every passage to the nothing zone with no previous condition unless a collapsed event gives that information to the next passage. The kineticmomentum energy negative oriented is interpreted as wave coming from an opposite fluctuation or opposite rotation. This can be a phase difference of $\pi$ at the cycle; when one is at manifest zone, the other solution is at nothing zone, no conflict of existence together as practical exclusion principle was presented by Wolfgang Pauli [19]. Both solutions with no preference for the next fluctuation after nothing passage (as position and direction) meanwhile conservation of fundamental values is maintain.

The negative mass-energy oriented was interpreted by Dirac as Antimatter, but it seems that the charge took more attention (greater force) than the energy dimension direction; Antimatter $\leftarrow \lambda \mathrm{Mo}=-\rightarrow \lambda \mathrm{Mo}$ as solutions 3 and 4 don't change on every fluctuation; information about type of mass is maintained during nothing passage. Quantum electrodynamics takes $\leftarrow \mathbf{C}$ as time going backwards inside the package. Fearless Dragan Hajdukovic [20] looks for gravitational implications of antimatter with matter. From gravitational view of hypothesis equation (13) where mass 1 is matter and mass 2 is antimatter, an attraction-repulsion is obtained; $\leftarrow \lambda \mathrm{M}_{2} v_{2}=\leftarrow \mathbf{C}=-\rightarrow \mathbf{C}$.

$$
\begin{aligned}
& \mathbf{F}_{1}=\mathrm{M}_{1} \gamma_{1} \mathrm{n}_{2}(-\lambda 2) \mathrm{Ghv}_{2}{ }^{2} /\left(\mathrm{C}^{3} \mathbf{R}^{2}\right)=\mathrm{M}_{1} \gamma_{1} \mathrm{n}_{2}(\leftarrow \mathbf{C}) \mathrm{G} \mathrm{h} v_{2} /\left(\mathrm{C}^{3}\right. \\
& \left.\mathbf{R}^{2}\right)=-\mathrm{M}_{1} \gamma_{1} \mathrm{n}_{2}(\rightarrow \mathbf{C}) \mathrm{G} \mathrm{h} v_{2} /\left(\mathrm{C}^{3} \mathbf{R}^{2}\right)(23) \\
& \mathbf{F}_{1}=-\mathrm{G} \mathrm{M}_{1} \gamma_{1}\left(\mathrm{n}_{2} \mathrm{~h} v_{2}\right) /\left(\mathrm{C}^{2} \mathbf{R}^{2}\right)=-\mathrm{G} \gamma_{1} \gamma_{2} \mathrm{M}_{1} \mathrm{M}_{2} \mathrm{C}^{2} /\left(\mathrm{C}^{\mathbf{2}} \mathbf{R}^{2}\right) \\
& \quad(24) \\
& \mathbf{F}_{1}=-\left(\mathrm{G} \gamma_{1} \gamma_{2}\right) \mathrm{M}_{1} \mathrm{M}_{2} / \mathbf{R}^{2}
\end{aligned}
$$

where $M_{1}$ matter with $M_{2}$ antimatter a repulsion force is deduced. This attraction-repulsion could be the reason why the universe expansion is so persistent. A conglomeration of matter masses that have an attraction between them but repels from the other group of antimatter masses $\mathbf{F}=$ $(-)(-)\left(G \gamma_{1} \gamma_{2}\right) M_{1} M_{2} / \mathbf{R}^{2}$ that attracts also between them. Cosmos expansion skillfully confirmed by Riess et al [21] 
and Perlmutter et al [22]. Also, a formation of antimatter or matter clusters is possible to be trapped by matter or antimatter surrounding where, if they have a balanced charge, the gravitational repulsion will maintain them encapsulated. Type of matter-antimatter prevails between existence cycle that attracts and repels such as the charge does.

\section{Conclusions}

The historic four core ideas of Quantum Theory and Theory of Relativity are: A) Energy in package of action h, containing uncertainty of simultaneous physical values. B) Existence as energy-momentum and space-time wave, duality of particle-waves. C) Probabilistic existence distributed in the package by the wave 15function; superposition of multiple existences with importance given by its probability. D) Mass as energy and gravitation as curved space. New hypothesis interprets those as: A) Cycle containing space presence in oscillating wave form, with inertial balance between manifest space and nothing zone with i math of complex numbers; energy wavelength as the 4 th or more dimensions, with 4 configurations between kinetic-momentum and mass energy. B) Existence with new space on every fluctuation, no full existence definition. Position-direction-spin-polarization, just obtaining new system valid values on new space. C) Cyclic existence with total energy and total momentum consolidated at nothing passage when space and their values are not present. Absolute conservation of total energy-momentumchargematter/type on all the cycle, including at nothing passage. D) Special Relativity flat space is interpreted as equivalent steady flow of space with the absence of energy. When energy interrelates with space gives an equivalent accelerated flow appreciated as gravitation and equivalent space flow speed gives a correction factor to constant G. Minkowski invariance as multidimensional energy with 3D space. Resuming, Theory of Space gives a joining view of 3D space with multidimensional space energy (helpful to $\mathrm{M}$ theory, String theory, Loop quantum gravity), where its oscillating manifestation values (proven quantum statistics) prevails in the absent space (nothing zone). Nothing is something.

\section{Conflicts of Interest}

The author declares no conflicts of interest regarding the publication of this paper.

\section{References}

[1] Born,M. 1926 Zur Quantenmechanik der Stoßvorg" ange Zeitschrift fur Physik 37 (12) 863-867

[2] Einstein, A. 1905 Zur Elektrodynamik bewegter Korper (On the Electrodynamics of Moving Bodies) Annalen der Physik 17891

[3] Einstein, A. 1915 Feldgleichungen der Gravitation (The Field Equations of Gravitation) Preussische Akademie der Wissenschaften, Sitzungsberichte 1915 (parte 2), 844-847
[4] Einstein, A. 1907 On the Relativity Principle and the Conclusions Drawn from It. Jahrbuch Der Radioaktivit" at und Elektronik 4 411-462 16

[5] Liberati S. and Maccione, L. 2014 Astrophysical Constraints on Planck Scale Dissipative Phenomena Phys. Rev. Lett. 112, 151301

[6] Jonsson, C. 1961 Elektroneninterferenzen an mehreren kunstlich hergestellten Feinspalten Zeitschrift f"ur Physik volume 161 454-474

[7] Bell, J.S. 1964 On the Einstein Podolsky Rosen Paradox Physics Physique Fizika 1195

[8] Hund, F. 1927 Zur Deutung der Molekelspektren. I Zeitschrift f'ur Physik 40 742-764

[9] Heisenberg, W. 1927 Uber den anschaulichen Inhalt der quantentheoretischen " Kinematik und Mechanik Zeitschrift f'ur Physik 43 (3-4) 172-198

[10] De Broglie, L. 1923 Waves and quanta Nature 112 (2815) 540

[11] Minkowski, H. 1907-1908 Die Grundgleichungen f'ur die elektromagnetischen Vorg"ange in bewegten K"orpern [The Fundamental Equations for Electromagnetic Processes in Moving Bodies] Nachrichten von der Gesellschaft der Wissenschaften zu G”ottingen Mathematisch-Physikalische Klasse 53111

[12] Lorentz, H.A. 1904 Electromagnetic phenomena in a system moving with any velocity smaller than that of light Proceedings of the Royal Netherlands Academy of Arts and Sciences 6 809-831

[13] Planck, M. 1900 Ueber das Gesetz der EnergieverteilungimNormalspectrum [On the law of the distribution of energy in the normal spectrum] Annalen der Physik 4(3) 553-563

[14] Schrodinger, E. 1926 Quantisierung als Eigenwertproblem Annalen der Physik 384 (4) 273 376

[15] Noether, E. 1918 Invariante Variationsprobleme Mathematisch, Physikalische Klasse 235-257

[16] Newton, I. 1687 Philosophiae Naturalis Principia Matematica, Joseph Streater - Royal Astronomy Society

[17] Dirac, P. 1928 The Quantum Theory of the Electron Proceedings of the Royal Society of London A 117 (778) 610-24

[18] Kaluza, T. 1921 Zum Unit“atsproblem in der Physik Sitzungsber. Preuss. Akad. Wiss. Berlin (Math. Phys.) 966-972 17

[19] Pauli, W. 1925 Uber den Zusammenhang des Abschlusses der Elektronengrup- " pen im Atom mit der Komplexstruktur der Spektren. Zeitschriftf"urPhysik. 31 (1): 765-783

[20] Hajdukovic, D. 2019 Antimatter gravity and the Universe Modern Physics Letters A 2030001

[21] Riess A.G. et al, 1998 Observational evidence from supernovae for an accelerating universe and a cosmological constant Astronomical Journal 116 (3) 1009-1038

[22] Perlmutter S. et al, 1999 Measurements of Omega and Lambda from 42 high redshift supernovae Astrophysical Journal 517 (2) 565-58 